\title{
Periodization in Cluster Dynamical Mean-Field Theory
}

\author{
M. Harland, I. Krivenko, A. I. Lichtenstein
}

$\mathrm{UH}$

\section{Introduction}

The Dynamical Mean Field Theory (DMFT) maps the lattice problem onto a single site coupled to an electron bath, i.e. the Mean Field. To include spatial correlations we use CDMFT, the cluster extension that connects a cluster of many correlated sites to an electron bath with a matrix-valued hybridization function. Whereas there is only one possible DMFT scheme for the single site, there are different schemes proposed for cluster calculations. They differ in the way they incorporate the lattice symmetry into the cluster calculation done by the impurity solver. We present a comparison of the self-energy and the cumulant periodization applying it to the 1-dimensional lattice as well as to the frustrated Kagome lattice. The CDMFT scheme we use is formulated in the real space. We solve the Hubbard cluster impurity model within a Hybridization Expansion Quantum Monte Carlo solver and compare the lattice Green's function and the local density of states to results of the Density Matrix Renormalization Group method.

\section{Methods}

Using DMFT (Georges et al., 1996) we map the Hubbard model

$$
H=\sum_{i j \sigma} t_{i j} c_{i \sigma}^{\dagger} c_{j \sigma}+\frac{U}{2} \sum_{i j} n_{i \uparrow} n_{i \downarrow}
$$

with the nearest neighbour hopping tensor $t_{i j}$ and the screened Coulomb interaction $U$ onto a multiorbital Anderson impurity model

$$
H_{A I M}=\sum_{\sigma} \varepsilon_{0} d_{\sigma}^{\dagger} d_{\sigma}+U n_{\uparrow} n_{\downarrow}+\sum_{k \sigma}\left(V_{k} c_{k \sigma}^{\dagger} d_{\sigma}+\text { H.c. }\right)+\sum_{k \sigma} \varepsilon_{k} c_{k \sigma}^{\dagger} c_{k \sigma}
$$

where the $c$ and $d$ operators act on bath and impurity fermions, respectively. $\varepsilon$ are kinetic energies, $V$ characterizes the hybridization and $U$ is the Coulomb repulsion only on the impurity. It describes the impurity coupled to a bath. We end up with a self-consistent set of equations:

$$
\begin{gathered}
S_{\text {eff }}=-\int_{0}^{\beta} d \tau \int_{0}^{\beta} d \tau^{\prime} \sum_{\sigma} c_{0 \sigma}^{\dagger}(\tau) \mathscr{G}_{0}^{-1}\left(\tau-\tau^{\prime}\right) c_{0 \sigma}\left(\tau^{\prime}\right)+U \int_{0}^{\beta} d \tau n_{0 \uparrow}(\tau) n_{0 \downarrow}(\tau), \\
\mathscr{G}_{0}\left(i \omega_{n}\right)^{-1}=i \omega_{n}+\mu-\Delta\left(i \omega_{n}\right), \\
G\left(\tau-\tau^{\prime}\right)=-<\mathrm{T}_{\tau} c(\tau) c^{\dagger}\left(\tau^{\prime}\right)>S_{\text {eff }} .
\end{gathered}
$$

$\mathscr{G}_{0}$ is the Weiss-field. It makes the quadratic part in the effective action $S_{e} f f . \Delta$ is the hybridization

$$
\Delta^{a b}\left(i \omega_{n}\right)=\sum_{k \alpha} V_{k}^{* a \alpha}\left(i \omega_{n}-\varepsilon_{k \alpha}\right)^{-1} V_{k}^{\alpha b}
$$

and $\mu$ the chemical potential. The integral of Eq. (5) is solved with a continous-time quantum MonteCarlo solver (Ferrero and Parcollet, 2013; Seth et al., 2013) using the hybridization expansion (Gull et al., 2011). Extending the theory to impurities of small clusters, the quantities of the DMFT equations becomes matrices over the cluster sites and we obtain the local Green's function summing over the sites of a superlattice, i.e. a lattice of clusters. In the case of Cellular-DMFT (C-DMFT) (Kotliar et al., 2001; Biroli et al., 2004) it modifies the self-consistency equations by

$$
\mathscr{G}^{0}\left(i \omega_{n}\right)^{-1}=\left(\frac{N_{c}}{N} \sum_{\tilde{k}} \frac{1}{i \omega_{n}+\mu-\varepsilon(\tilde{k})-\Sigma_{c}\left(i \omega_{n}\right)}\right)^{-1}+\Sigma_{c}\left(i \omega_{n}\right) .
$$

$c$ stands for ćlusteránd $\tilde{k}$ is a reciprocal lattice vector of the reduced Brillouin zone. To incorporate the lattice symmetries in our solution we periodize the cluster results

$$
Q_{l a t}^{P}\left(i \omega_{n}, k\right)=\frac{1}{N_{c}} \sum_{i j=1}^{N_{c}} e^{i k\left(r_{i}-r_{j}\right)} Q_{c}\left(i \omega_{n}\right) i j
$$

The choice of $Q$ is not unique. We use the self-energy $Q=\Sigma$ and the cumulant $Q=M$ $\left(i \omega_{n}+\mu-\Sigma\right)^{-1}$. Improved estimators have been proposed (Biroli and Kotliar, 2002). They read

$$
Q_{l a t}^{W}\left(i \omega_{n}, k\right)=\sum_{i, j=1}^{N_{c}} w_{i j} e^{i k\left(r_{i}-r_{j}\right)} Q_{c}\left(i \omega_{n}\right)_{i j} .
$$

These methods are proven to be manifestly causal. A further method has been proposed (Lichtenstein and Katsnelson, 2000) that could not be proven to be causal. It treats the self-energy as a renormalization of the local hopping within the Tight-Binding frame

$$
Q_{l a t}^{T B}\left(i \omega_{n}, k\right)=\sum_{\tilde{r}} e^{i \tilde{k} \tilde{r}} Q_{\tilde{r}}^{T B}\left(i \omega_{n}\right) .
$$

$Q_{\tilde{r}}^{T B}$ has to be set up analogous to the intercluster-hopping $t_{r}$ with elements of $Q_{c}$

\section{References}

Biroli, G. and Kotliar, G. (2002). Cluster methods for strongly correlated electron systems. Phys. Rev. $B, 65: 155112$.

Biroli, G., Parcollet, O., and Kotliar, G. (2004). Cluster dynamical mean-field theories: Causality and classical limit. Phys. Rev. B, 69:205108.

Ferrero, M. and Parcollet, O. (2013). TRIQS: a Toolbox for Research in Interacting Quantum Systems. Georges, A., Kotliar, G., Krauth, W., and Rozenberg, M. J. (1996). Dynamical mean-field theory of strongly correlated fermion systems and the limit of infinite dimensions. Rev. Mod. Phys., 68:13-125. Gull, E., Millis, A. J., Lichtenstein, A. I., Rubtsov, A. N., Troyer, M., and Werner, P. (2011) Continuous-time monte carlo methods for quantum impurity models. Rev. Mod. Phys., 83:349-404. Hafermann, H. (2010). Numerical Approaches to Spatial Correlations in Strongly Interacting Fermion Systems. Cuvillier Verlag Gttingen.

Kotliar, G., Savrasov, S. Y., Pálsson, G., and Biroli, G. (2001). Cellular dynamical mean field approach to strongly correlated systems. Phys. Rev. Lett., 87:186401.

Lichtenstein, A. and Katsnelson, M. (2000). Antiferromagnetism and d-wave superconductivity in cuprates: A cluster dynamical mean-field theory. Phys. Rev. B, 62:R9283-R9286.

Seth, P., Krivenko, I., Ferrero, M., and Parcollet, O. (2013). Triqs fast hybridization-expansion solver to be published.

\section{2-Site Cluster - 1D Lattice}
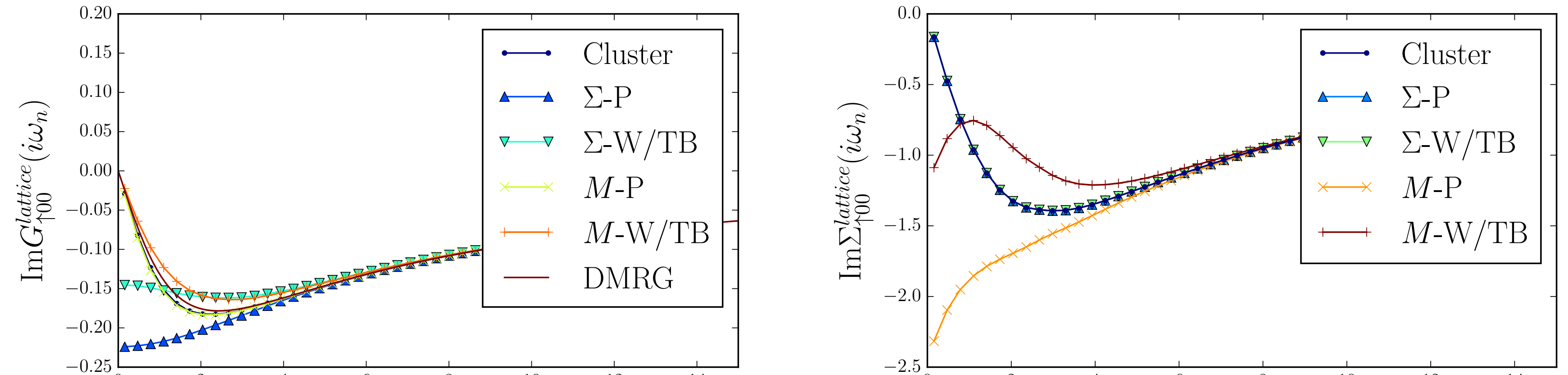

We compare the different periodization schemes for the 2-site cluster within the 1D lattice with DMRG results taken from Hafermann (2010). For $Q_{l a t}^{W}$ we use (Biroli and Kotliar, 2002)

$$
w=\left(\begin{array}{cc}
\frac{1}{2} & 1 \\
1 & \frac{1}{2}
\end{array}\right)
$$

which leads to the same result as for $Q_{l a t}^{T B}$ where we defined

$$
Q_{\tilde{r}}\left(i \omega_{n}\right)=\left\{\begin{array}{lll}
Q_{0}\left(i \omega_{n}\right)=\frac{Q_{00}^{c}\left(i \omega_{n}\right)+Q_{11}^{c}\left(i \omega_{n}\right)}{2} & \text { for } & \tilde{r}=(0,0) \\
Q_{1}\left(i \omega_{n}\right)=\frac{Q_{01}^{c}\left(i \omega_{n}\right)+Q_{10}^{c}\left(i \omega_{n}\right)}{2} & \text { for } \quad \tilde{r} \in\{(1,0),(-1,0)\}
\end{array}\right.
$$

where $Q_{0}$ and $Q_{1}$ are the on-site and the nearest-neighbour quantity, respectively. The lattice results are then

and

$$
\begin{gathered}
Q_{l a t}^{P}\left(i \omega_{n}, k\right)=Q_{0}\left(i \omega_{n}\right)+Q_{1}\left(i \omega_{n}\right) \cos (2 \pi k) \\
Q_{l a t}^{W / T B}\left(i \omega_{n}, k\right)=Q_{0}\left(i \omega_{n}\right)+2 Q_{1}\left(i \omega_{n}\right) \cos (2 \pi k) .
\end{gathered}
$$

$Q_{l a t}^{W / T B}\left(i \omega_{n}, k\right)=Q_{0}\left(i \omega_{n}\right)+2 Q_{1}\left(i \omega_{n}\right) \cos (2 \pi k)$

Numerical results for the local lattice Green's function (left) and the local lattice self-energy (right) are shown above. Parameters are listed below.

$$
\beta \quad t \quad U \mu \text { nr. of } k \text {-pts. }
$$

$\begin{array}{lllll}20 & -1 & 6 & 3 & 128\end{array}$

\section{3-Site Cluster - Kagome Lattice}

The Kagome lattice is subject of recent research due to its geometrical frustration. It is assumed to have a spin liquid ground state, that may serve as an explanation for high-temperature superconductivity. The state is highly degenerated and competes with antiferromagnetic order at low temperatures. Real materials with this structure show heavy fermion behavior without having f-electrons, but only $\mathrm{d}$ electrons.
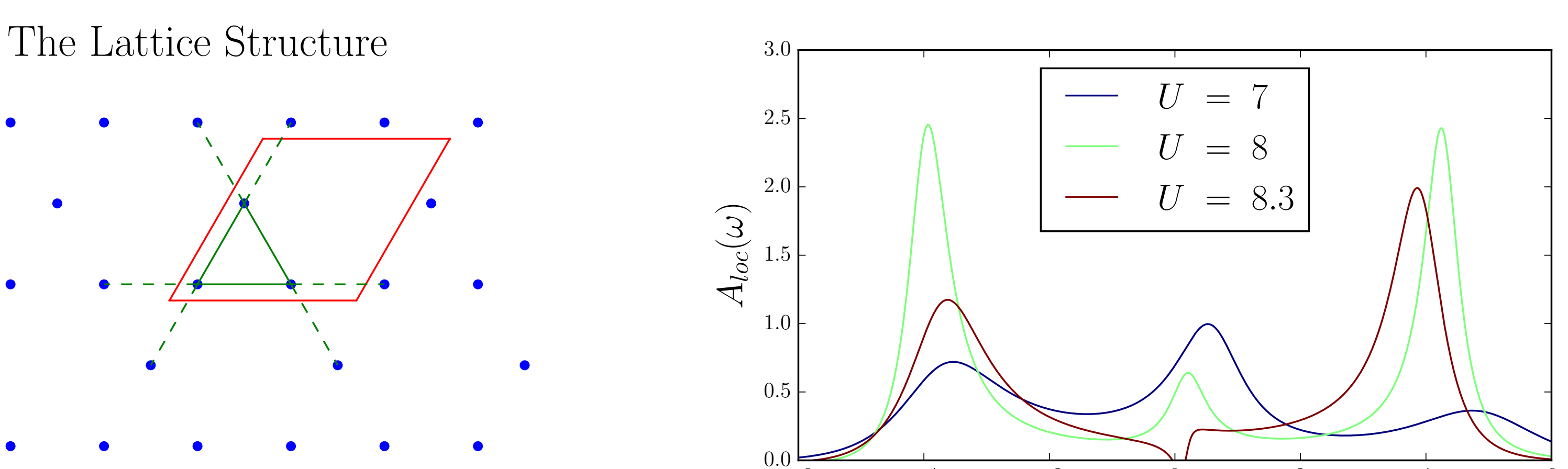

The structure of the Kagome lattice is shown (left) and the Mott transition with quasi-particle coeherence peak, too (right). $M^{T B}$-Periodization has been used. Analytical continuation has been done using the Pade-approximation. Parameters are listed below.

$$
\begin{array}{ccccc}
\beta & t & \mu & \text { nr. of } \tilde{k} \text {-pts. } \\
10 & -1 & \text { half filling } & 32
\end{array}
$$
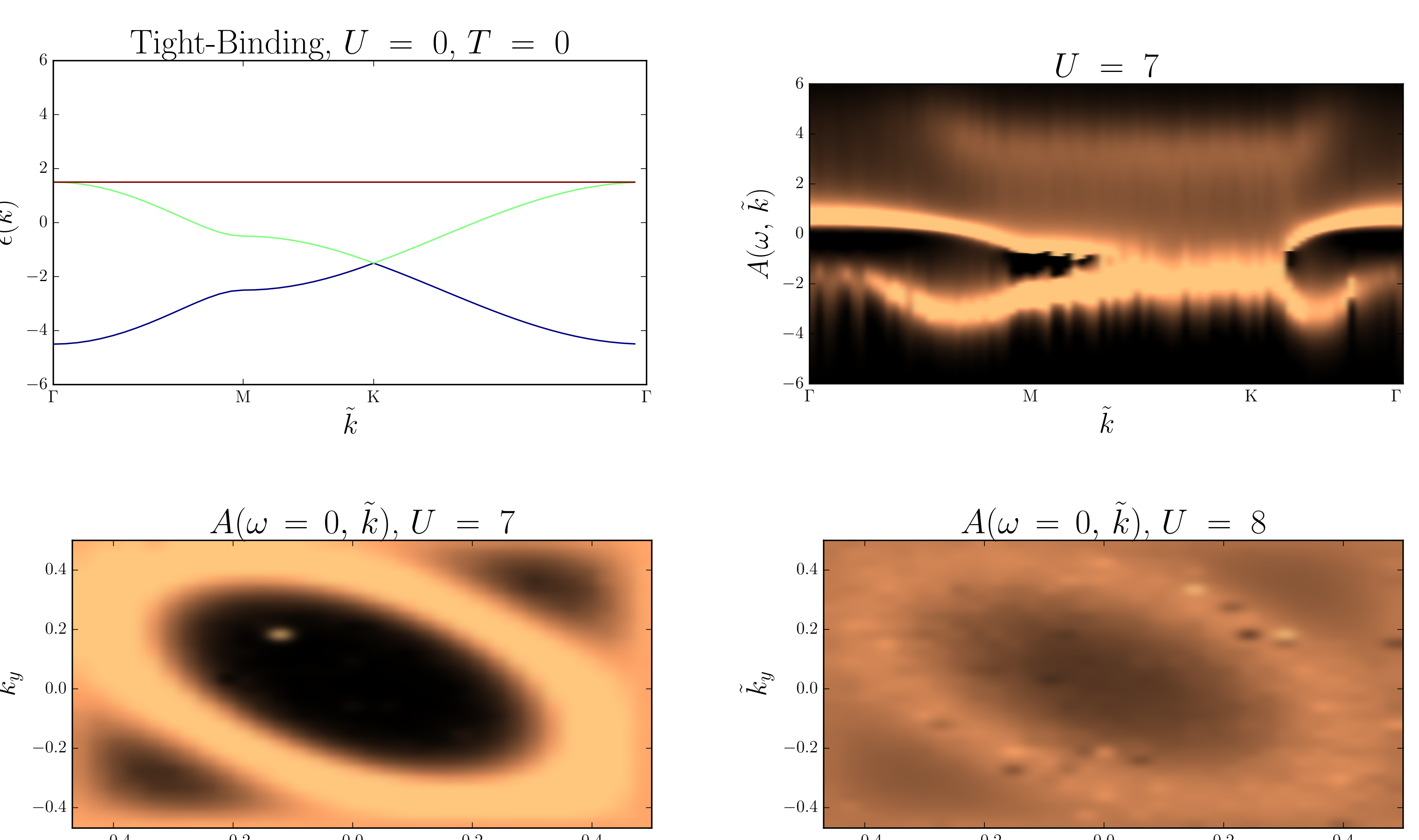

The Tight-Binding bandstructure (upper left) and $\tilde{k}$-resolved spectral functions for $U=7$ (lower left) and $U=8$ (right) are shown. The solutions are paramagnetic as expectet at high temperatures. We observe a flattening of the bands close to the Mott-transition. The states become strongly localized before the vanish near the Fermi-energy, due to strong Coulomb repulsion. We explain the local ore they vais necalization of states by strong fluctuations from the large temperature that makes scattering processes incoherent and thus prevents electrons from hopping. 